\title{
Frittage de YBaCuO, implications du diagramme de phases
}

\author{
M. Gervais, A. Douy, B. Dubois, J. P. Coutures et P. Odier \\ Centre de Recherches sur la Physique des Hautes Températures, CNRS, 45071 Orléans Cedex 2, France
}

(Reçu le 9 décembre 1988, révisé et accepté le 17 février 1989)

\begin{abstract}
Résumé. - Les motivations de ce travail sont d'examiner les implications du diagramme de phases du système pseudo-ternaire $\mathrm{Y}_{2} \mathrm{O}_{3}$-CuO-BaO sur le frittage de composés proches de la composition supraconductrice $\mathrm{YBa}_{2} \mathrm{Cu}_{3} \mathrm{O}_{7-p}$. Dans ce travail préliminaire, on s'est intéressé à mettre en évidence les transformations solide $\rightarrow$ liquide de ce système, au voisinage de la composition (123). Deux transformations apparaissent à 915 et $935^{\circ} \mathrm{C}$, selon la composition du produit, elles jouent un rôle important sur le frittage et l'homogénéité chimique du produit fritté. Il ne semble pas y avoir de transformations liquides dans le domaine (123)-(211)$\mathrm{BaCuO}_{2}$, il en résulte une homogénéité chimique plus grande des échantillons frittés.
\end{abstract}

\begin{abstract}
The motivations of this experimental work are to underline the implications between the phases diagram constitution and the sintering of $\mathrm{YBaCuO}$ superconductors. This preliminary work is focussed on the solid $\rightarrow$ liquid transformations of this system, in the vicinity of the (123) phase. Two transformations are observed at 915 and $935^{\circ} \mathrm{C}$ depending of the composition of the compound. They both have an important role on the sintering process and the chemical homogeneity of the ceramic. No such transformations seems to occur in the domain (123)-(211)- $\mathrm{BaCuO}_{2}$, the sintered sample has therefore a better chemical homogeneity.
\end{abstract}

\section{Introduction.}

L'utilisation des oxydes supraconducteurs à l'état massif est subordonnée à l'élaboration de céramiques denses, chimiquement homogènes et en particulier à l'obtention de joints de grains exempts de phases intergranulaires non supraconductrices. Cette contrainte est particulièrement critique en raison de la faible longueur de cohérence caractérisant ce matériau [1, 2]. L'élimination des phases secondaires nécessite de maîtriser deux étapes importantes de l'élaboration des céramiques : i) la synthèse de poudres très homogènes en composition et aptes au frittage; ii) le frittage de ces poudres dans des conditions évitant le développement d'hétérogénéités chimiques. Le frittage des poudres d'YBaCuO est contrôlé par l'apparition d'une phase liquide vers 910-940 ${ }^{\circ} \mathrm{C}$ [3-5], qui est à l'origine de phases secondaires intergranulaires. La constitution du diagramme de phases joue un rôle déterminant pour prévoir les effets du frittage sur l'homogénéité chimique. L'objectif de cet article est de montrer d'une part cette relation et d'autre part d'élargir la connaissance relative au diagramme de phases en vue de mieux maîtriser le frittage des oxydes supraconducteurs.
Deux méthodes de synthèse de poudres à partir de précurseurs liquides ont été mises au point qui privilégient une répartition très homogène des cations, une basse température d'obtention et une morphologie adaptée au frittage. Ces poudres ont été utilisées pour aborder l'étude du frittage et du diagramme de phases pour laquelle nous donnerons des résultats préliminaires. Ils concernent plus particulièrement l'existence de phases liquides, à des températures inférieures à $950^{\circ} \mathrm{C}$, sur les lignes de conjugaison et dans les triangles de compatibilité faisant intervenir le composé $\mathrm{YBa}_{2} \mathrm{Cu}_{3} \mathrm{O}_{7-x}$ (123). Ils sont corrélés avec les données issues du frittage de $\mathrm{YBa}_{2} \mathrm{Cu}_{3} \mathrm{O}_{7-p}$ (échantillon c) et de deux types d'échantillons de compositions proches de (123), respectivement (a): $\mathrm{Y}_{1-x} \mathrm{Ba}_{2-y} \mathrm{Cu}_{3+z} \mathrm{O}_{7-p}$, $\mathrm{Y}_{1+x} \mathrm{Ba}_{2+y} \mathrm{Cu}_{3-z} \mathrm{O}_{7-p}$.

\section{Méthode expérimentale.}

1.1 SYNTHÈSE DES POUDRES. - Deux méthodes ont été mises en œuvre, toutes deux partant de précurseurs liquides. Dans le premier cas, méthode I, on gélifie in situ une solution précurseur par un polymère organique très classique dans le domaine des sciences biologiques : le gel de polyacrylamide. 
La solution précurseur est obtenue par dissolution en milieu aqueux, additionné d'acide citrique, de nitrate d'yttrium, de carbonate de baryum et d'acétate de cuivre dans les proportions requises. Le $\mathrm{pH}$ est fixé à 7 par addition d'ammoniaque. Le précurseur gélifié est parfaitement stable et possède une couleur bleue. Le gel est calciné dans un four programmable ventilé. Dès $800^{\circ} \mathrm{C}$ sous air, on obtient une poudre fine d'YBaCuO (5-10 $\left.\mathrm{m}^{2} \mathrm{~g}^{-1}\right)$, formée d'agglomérats tendres. La poudre contient encore des traces de $\mathrm{BaCO}_{3}$ et $\mathrm{YBa}_{2} \mathrm{Cu}_{3} \mathrm{O}_{7-p}$ à la structure quadratique, même après un refroidissement lent. Ce phénomène a déjà été remarqué dans la synthèse par la voie oxalate $[6,7]$. Par contre un recuit à $860^{\circ} \mathrm{C}$ dans l'air, suivi d'un refroidissement lent conduit à la phase orthorhombique aux propriétés supraconductrices [8]. Cette méthode présente le grand intérêt d'être directe, facile à mettre en œuvre et se prête à la synthèse des diverses combinaisons nécessaires à l'établissement d'un diagramme de phases. La deuxième méthode, II, vise l'obtention de particules sphériques et de granulométrie ajustée. Elle consiste en la décomposition thermique contrôlée d'un brouillard précurseur d'YBaCuO obtenu par ultra-sons [9]. Le précurseur liquide est identique à celui utilisé dans la méthode $\mathrm{I}$. Dès $835^{\circ} \mathrm{C}$, on forme la phase $\mathrm{YBaCuO}$ qu'un refroidissement lent rend orthorhombique avec les propriétés supraconductrices recherchées [10]. Les particules sont sphériques et ont un diamètre moyen de $1,7 \mu \mathrm{m}$. Celui-ci peut être ajusté en jouant sur la fréquence des ultra-sons et/ou la dilution du précurseur [11]. Ces particules sont en fait des agglomérats de cristallites d'environ $100 \mathrm{~nm}$ dont la dimension augmente à $250 \mathrm{~nm}$ par calcination à $890^{\circ} \mathrm{C}$, elles ont vraisemblablement la forme de plaquettes.

1.2 Diagramme DE PHASES. - L'étude du diagramme de phases du système peudo-ternaire $\mathrm{Y}_{2} \mathrm{O}_{3}-\mathrm{BaO}-\mathrm{CuO}$ a été abordée en utilisant principalement l'analyse thermique différentielle (ATD) et la diffraction des rayons $\mathrm{X}$ à température ambiante. L'ATD a permis de déterminer les transitions de phases jusqu'à $1300^{\circ} \mathrm{C}$, les mesures ont été faites sous air avec une vitesse de montée en température fixée à $600^{\circ} \mathrm{C} \mathrm{h}^{-1}$. La microstructure des échantillons a été observée au microscope à balayage. L'étude du diagramme de phases est compliquée par l'existence de réactions matériau-support. Ces réactions s'intensifient en présence de phases liquides. Le baryum réagit avec le platine et l'alumine et le cuivre avec le platine. Il a donc été nécessaire de trouver un matériau compatible. Nous avons utilisé avec de très bons résultats des creusets de zircone yttriée (3 mole \%), totalement denses, élaborés au laboratoire.

1.3 Frittage. - L'étude du frittage a été réalisée sur les poudres obtenues par le mode II et compac- tées à sec en isostatique. Le taux de compaction ainsi atteint correspond à environ $60 \%$ de la densité théorique. On a étudié : i) la cinétique de retrait avec un dilatomètre différentiel $\left({ }^{1}\right)$; ii) le mode de croissance granulaire par examen en microscopie à balayage des fractures d'échantillons frittés ; iii) l'apparition d'hétérogénéités chimiques résultant du traitement thermique, par microsonde de Castaing. Dans ce dernier cas, chaque échantillon est soumis à 100 points d'analyse (quelques $\mu \mathrm{m}^{3}$ ) régulièrement espacés de $100 \mu \mathrm{m}$.

\section{Résultats et discussion.}

2.1 FRITTAGE. - Le frittage d'échantillons compactés à sec, nécessite d'atteindre au moins $930^{\circ} \mathrm{C}$ pour que la densification soit achevée. Dans ces conditions, les poudres élaborées par la méthode II conduisent à une densité supérieure à $90 \%$ de la densité théorique. Une diminution de taille des agglomérats de la poudre (de $2 \mu \mathrm{m}$ à $0,5 \mu \mathrm{m}$ ) n'a qu'un effet négligeable sur la température de fin de frittage, définie par l'arrêt du retrait lors d'une montée en température à vitesse constante. Ceci indique que les mécanismes classiques de transport en phase solide sont court-circuités, selon toute vraisemblance, par un transport en phase liquide. $\mathrm{Ce}$ phénomène est bien montré par l'accélération brutale du retrait vers $900-930{ }^{\circ} \mathrm{C}$ que l'on observe sur les courbes dilatométriques de frittage $[9,16,17]$ (Fig. 1). De même l'examen de l'évolution des microstructures entre $900^{\circ}$ et $930^{\circ} \mathrm{C}$ est conforme à ce mécanisme : la croissance granulaire est dans cette gamme de température extrêmement rapide et les fractures présentent des faciès à l'aspect amorphe [9].

Il faut noter cependant que de très légers écarts sur les rapports cationiques entraînent des différences dans le comportement au frittage et dans l'homogénéité chimique des céramiques obtenues. Ainsi sur la figure 1 est représenté le retrait au frittage de deux compositions; i.e.

(a) :

$$
\mathrm{Y}_{1-x} \mathrm{Ba}_{2-y} \mathrm{Cu}_{3+z} \mathrm{O}_{7-p}
$$

et

(b) :

$$
\mathrm{Y}_{1+x} \mathrm{Ba}_{2+y} \mathrm{Cu}_{3-z} \mathrm{O}_{7-p} .
$$

On constate que le frittage du composé (b) est plus lent que celui de (a) ; corrélativement la température de fin de frittage de (b) est plus élevée, de $40^{\circ} \mathrm{C}$. La microstructure de ces échantillons est différente : alors que l'échantillon (a), comme d'ailleurs la composition (123), a une microstructure caractéristique d'un frittage en phase liquide; au contraire,

(1) Dilatomètre SETARAM-crpht, BP 34, 69641 Caluire Cedex, France. 


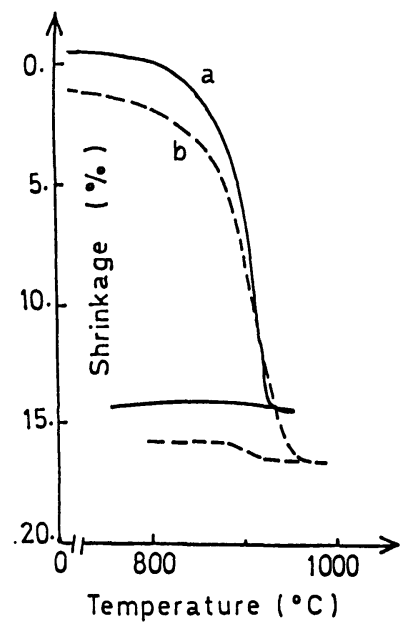

Fig. 1. - Retrait au frittage de deux compositions de $\mathrm{YBaCuO}:$ (a) $\mathrm{Y}_{1-x} \mathrm{Ba}_{2-y} \mathrm{Cu}_{3+z} \mathrm{O}_{7-p}$, (b) $\mathrm{Y}_{1+x} \mathrm{Ba}_{2+y}$ $\mathrm{Cu}_{3-z} \mathrm{O}_{7-p}$.

[Shrinkage versus $T$ during the sintering of two compositions of $\mathrm{YBaCuO}$ : $\left.\mathrm{Y}_{1+x} \mathrm{Ba}_{2+y} \mathrm{Cu}_{3-z} \mathrm{O}_{7-p} \cdot\right]$

(a) $\mathrm{Y}_{1-x} \mathrm{Ba}_{2-y} \mathrm{Cu}_{3+z} \mathrm{O}_{7-p}$

l'échantillon (b) présente des fractures beaucoup plus franches, ce qui laisse supposer qu'il a peu subi l'action de phases liquides.

La comparaison des analyses chimiques ponctuelles effectuées sur les échantillons (a), (b) et sur un échantillon (c) de composition $\mathrm{Yba}_{2} \mathrm{Cu}_{3} \mathrm{O}_{7-p}$, figure 2 , montre une homogénéité chimique sensiblement mieux conservée dans l'échantillon (b) que dans les échantillons (a) et (c). L'ensemble de ces différences qui sont significatives sont liées à la nature et à la quantité de phases liquides se développant dans l'échantillon au cours de son frittage.

2.2 DiAgRAMME DE PHASES. - Afin d'accéder à plus d'informations sur l'origine de ces phases liquides, une étude du diagramme de phases est nécessaire. Dans cette première approche, on a cherché à mettre en évidence les transformations solide $\rightarrow$ liquide. Les compositions étudiées sont représentées sur le diagramme établi à $950{ }^{\circ} \mathrm{C}$, initialement par Roth et al. [12] et précisé par d'autres auteurs [1315]. Ces compositions se localisent principalement dans les triangles de compatibilité centrés autour de (123) : (123)- $\mathrm{BaCuO}_{2}-\mathrm{CuO}, \quad$ (123)-CuO-(211) et (123)-(211)- $\mathrm{BaCuO}_{2}$. De nombreux échantillons ont été préparés par synthèse, méthodes I et II, ils sont notés $(\boldsymbol{\Delta})$ dans la figure 3 . Les autres compositions, notées (O), ont été obtenues par réaction à l'état solide entre des composés synthétisés puis mélangés dans les proportions appropriées.

Le composé supraconducteur (123) présente successivement trois transitions endothermiques lorsqu'il est chauffé à l'air jusqu'à $1300^{\circ} \mathrm{C}$. Une première transition de faible intensité est observée à $935^{\circ} \mathrm{C}$. Une seconde transition nettement plus

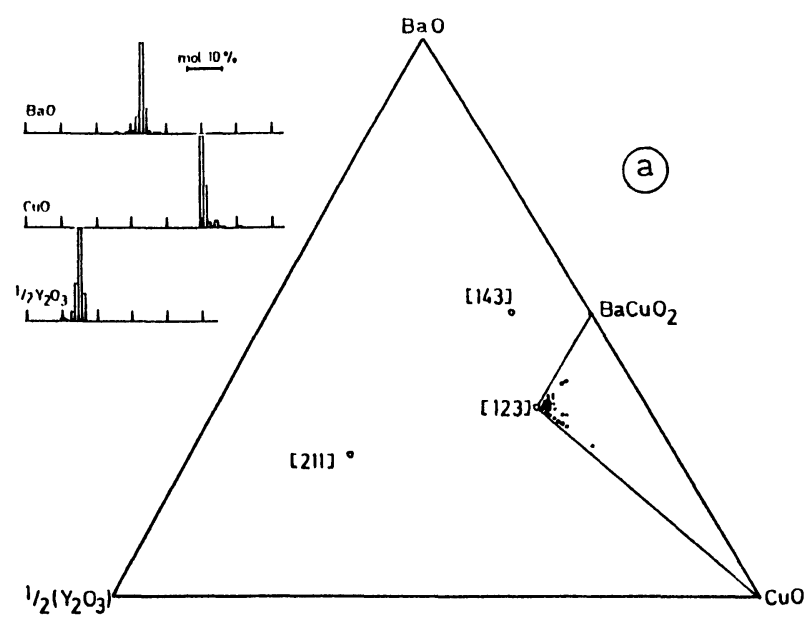

a)

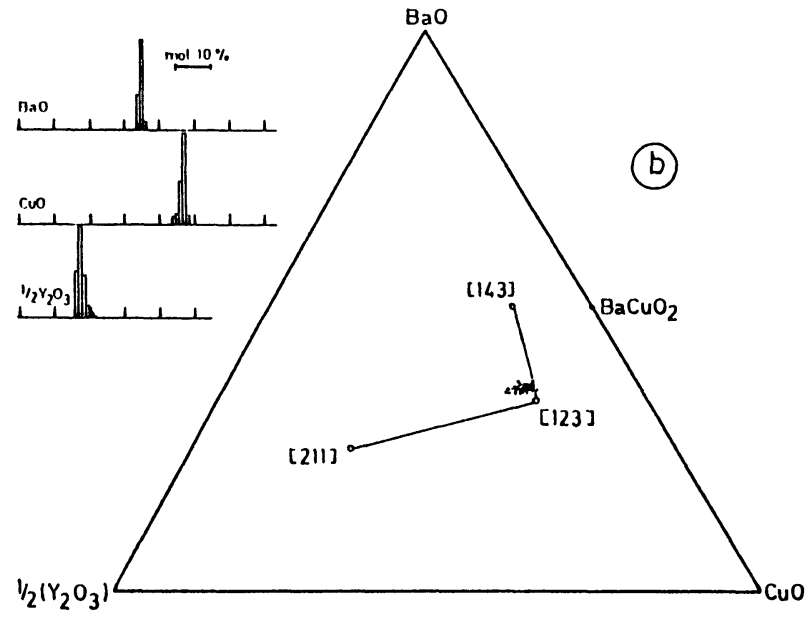

b)

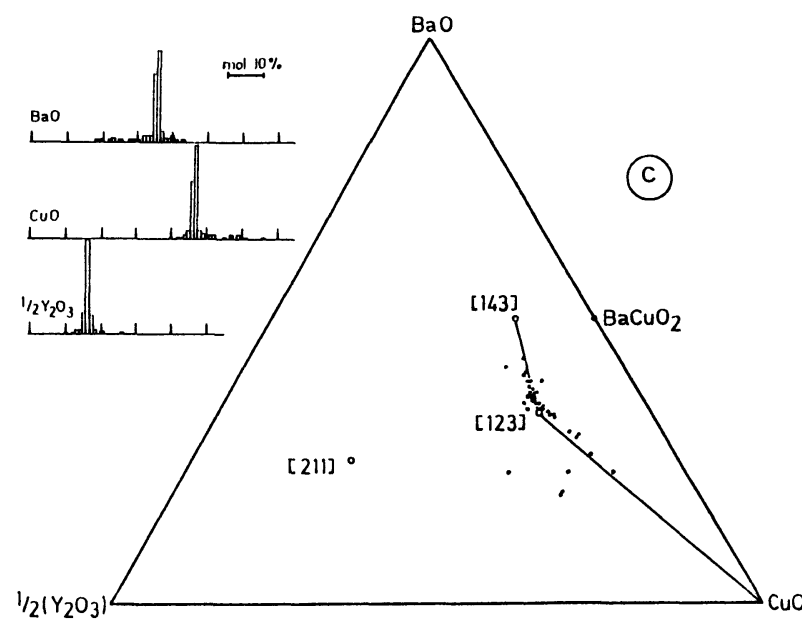

c)

Fig. 2. - Analyses chimiques ponctuelles de trois échantillons frittés (a), (b) et (c): $\mathrm{YBa}_{2} \mathrm{Cu}_{3} \mathrm{O}_{7-p}$.

[Punctual chemical analysis of three sintered samples (a), (b) and (c) : $\mathrm{YBa}_{2} \mathrm{Cu}_{3} \mathrm{O}_{7-p}$. ] 


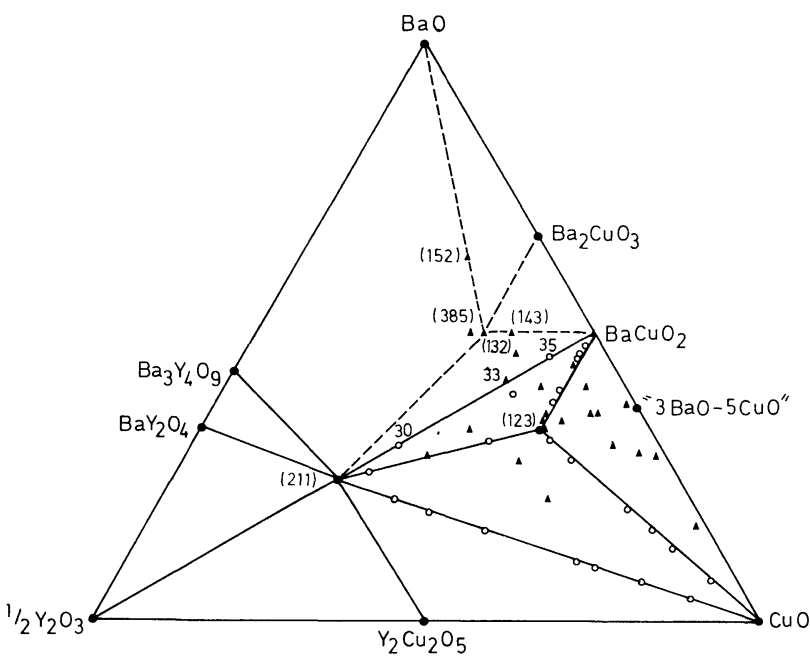

Fig. 3. - Diagramme de phases pseudo-ternaire $\mathrm{Y}_{2} \mathrm{O}_{3}$ $\mathrm{BaO}-\mathrm{CuO}$, établi à $950^{\circ} \mathrm{C}$, selon [12-15]. Les points $(\boldsymbol{\Delta})$ et (O) représentent les compositions étudiées dans ce travail : (A) compositions obtenues par synthèse selon les méthodes I et II, (O) compositions obtenues par réaction à l'état solide.

[Isothermal section of the pseudo-ternary phase diagram of $\mathrm{Y}_{2} \mathrm{O}_{3}-\mathrm{BaO}-\mathrm{CuO}$ at $950{ }^{\circ} \mathrm{C}$ according to [12-15]. The points $(\boldsymbol{\Delta})$ and $(\mathrm{O})$ are compositions studied in this work : (A) synthesized according to method I and II, (O) obtained by solid state reaction.]

intense est détectée à $995^{\circ} \mathrm{C}$ : elle correspond à la fusion incongruente du composé et à sa décomposition en (211) plus un liquide. Une troisième transition se produit à $1240^{\circ} \mathrm{C}$, elle correspond à la fusion péritectique du composé (211) qui se décompose alors en $\mathrm{Y}_{2} \mathrm{O}_{3}+$ liquide. La transition à $935^{\circ} \mathrm{C}$ est corrélée avec une perte de masse significative [9] (départ d'oxygène), elle conditionne pour beaucoup le frittage du composé $\mathrm{YBa}_{2} \mathrm{Cu}_{3} \mathrm{O}_{7-p}$ et son homogénéité chimique, voir figure 2 et référence [18]. L'étude par diffraction aux rayons $\mathrm{X}$ de produits trempés à partir de températures comprises entre 900 et $990^{\circ} \mathrm{C}$ ne permet pas de conclure à la présence de nouvelles phases. Cependant l'observation en microscopie à balayage (sur poudres et frittés) montre clairement la présence de zones ayant partiellement fondues. Cette transition à $935^{\circ} \mathrm{C}$ apparaît sur la ligne de conjugaison (123)- $\mathrm{BaCuO}_{2}$ jusqu'à des teneurs en (123) voisines de $67 \%$, sur la ligne (123)-(211) jusqu'à des teneurs en (123) proches de $1 \%$ et sur la ligne (123)-CuO, elle apparaît jusqu'à des teneurs de $6 \%$ en (123). Cette transformation apparaît aussi dans le triangle (123)-CuO(211), l'ensemble de ces données est résumé dans la figure 4.

Plusieurs coupes entre le composé (123) et différentes compositions du système binaire $\mathrm{BaCuO}_{2}$ $\mathrm{CuO}$ ont été étudiées. Un exemple des résultats obtenus pour la coupe (123)/3 $\mathrm{BaO}-5 \mathrm{CuO}$ est repré-

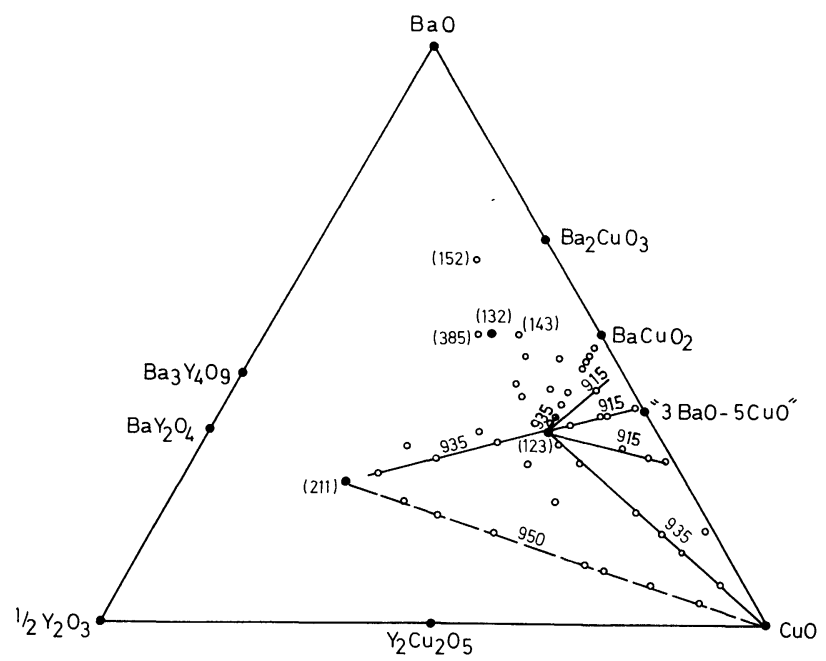

Fig. 4. - Régions où des phases liquides sont détectées autour du composé supraconducteur (123). Leurs températures d'apparition pour $900<T<995^{\circ} \mathrm{C}$ sont indiquées.

[Areas where liquid phases are detected around the superconductor phase (123). Their temperature are reported for the range $900-995^{\circ} \mathrm{C}$.]

senté sur la figure 5. La diffraction des rayons $X$ d'échantillons calcinés à $900^{\circ} \mathrm{C}$ (l'équilibre étant atteint dès cette température grâce à la bonne réactivité des poudres) montre la coexistence de composés (123), $\mathrm{BaCuO}_{2}$ et $\mathrm{CuO}$ dans tout le domaine de compositions. On observe une transition endothermique à $915^{\circ} \mathrm{C}$, jusqu'à des teneurs en (123) aussi faibles que $2 \%$, cette transformation a aussi été notée sur la figure 4 . C'est cette transition qui est responsable de l'accélération du frittage dans ce domaine. Cette transition correspond à l'apparition d'une phase liquide, sans disparition du composé (123). L'invariance de la température de cette transition pour toutes les compositions étudiées laisse penser qu'il s'agit de la fusion d'un eutectique ternaire, très pauvre en yttrium, dont la composition reste à préciser.

A l'intérieur du triangle de compatibilité (123)(211) $-\mathrm{BaCuO}_{2}$, les compositions étudiées n'ont pas révélé de transitions pour des températures inférieures à $990^{\circ} \mathrm{C}$ où apparaît la décomposition péritectique de (123). Cette observation est en parfait accord avec une homogénéité chimique plus grande des échantillons frittés.

\section{Conclusion.}

Cette étude préliminaire du diagramme de phases du système pseudo-ternaire $\mathrm{Y}_{2} \mathrm{O}_{3}-\mathrm{CuO}-\mathrm{BaO}$ permet d'identifier l'origine probable des phases liquides contrôlant pour une grande part le frittage de compositions proches de $\mathrm{YBaCuO}$ (123).

Les échantillons présentant un déficit en yttrium : i.e. $\mathrm{Y}_{1-x} \mathrm{Ba}_{2-y} \mathrm{Cu}_{3+z} \mathrm{O}_{7-p}$ sont soumis à une transi- 


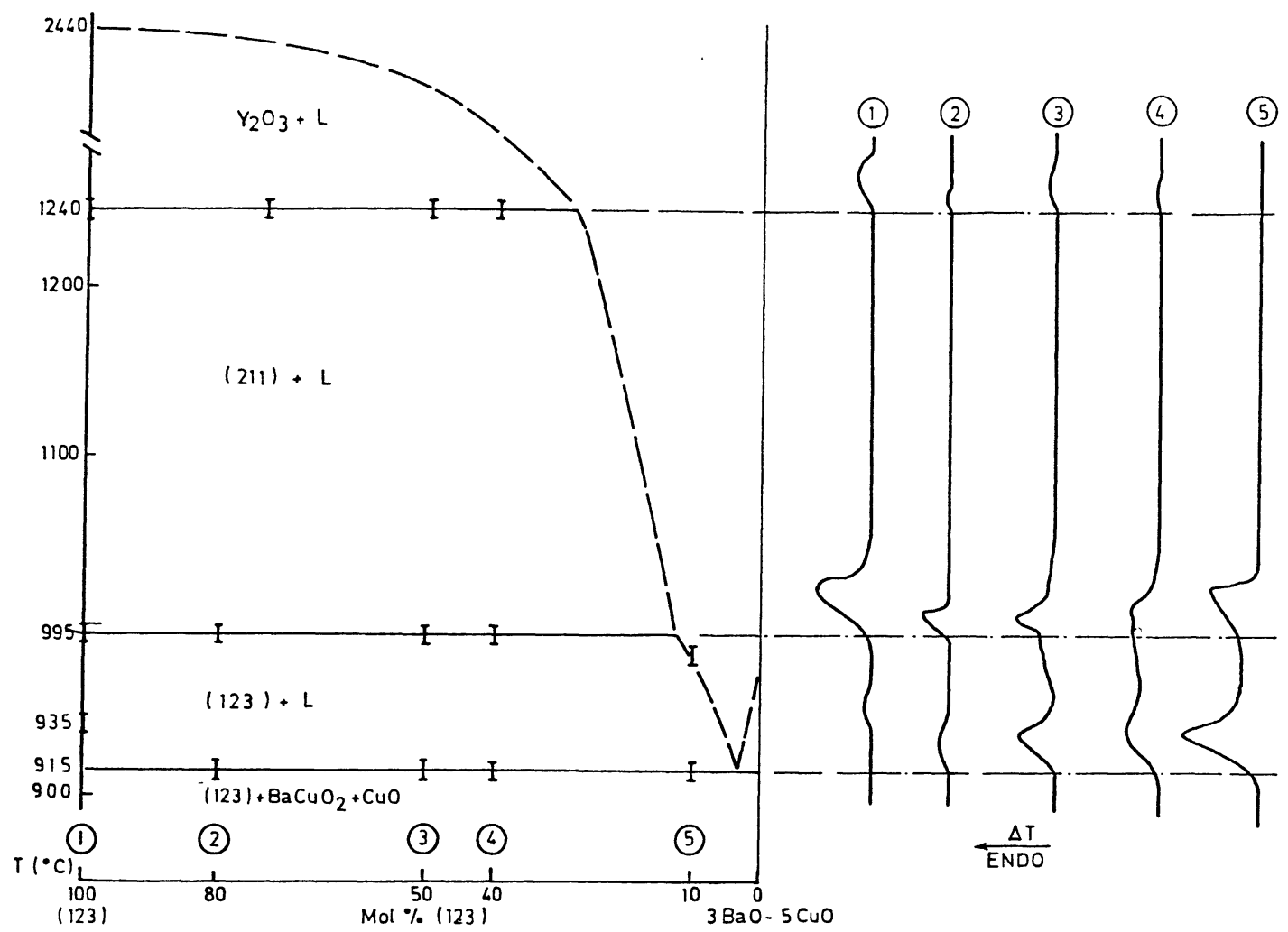

Fig. 5. - Coupe pseudo-binaire (123)-« $3 \mathrm{BaO}-5 \mathrm{CuO} »$ étudiée par ATD et diffraction des rayons X. Les températures sont prises à la montée.

[Pseudo-binary section (123)-« $3 \mathrm{BaO}-5 \mathrm{CuO} »$ obtained by DTA and $\mathrm{X}$ ray diffraction. The points are measured during the heating.]

tion liquide à $915^{\circ} \mathrm{C}$ qui résulte de la fusion d'un eutectique ternaire du triangle (123)- $\mathrm{CuO}-\mathrm{BaCuO}_{2}$ très pauvre en yttrium.

Cette transformation ne doit pas être confondue avec celle à $935^{\circ} \mathrm{C}$ apparaissant dans le composé stœchiométrique.
Il ne semble pas y avoir de transformations liquides en dessous de $995^{\circ} \mathrm{C}$ dans le domaine (123)(211) $-\mathrm{BaCuO}_{2}$. Les frittés de compositions appartenant à ce domaine et proches de la phase (123), paraissent chimiquement plus homogènes que dans d'autres régions.

\section{Bibliographie}

[1] Bednorz J. and Muller K. A., Rev. Mod. Phys. 60 (1988) 585.

[2] Forro L., Raki M., Ayache C., Stamp P. C. E., Henry J. Y., Rossat-Mignot J., Physica $C$ 153-155 (1988) 1357.

[3] Rahaman M. N., De Jonghe L. C. and Chu M. Y., J. Am. Ceram. Soc. 71 (1988) C237.

[4] Sarikaya M. and Theil B. L., J. Am. Ceram. Soc. 71 (1988) C305.

[5] Guha J. P., J. Am. Ceram. Soc. 71 (5) (1988) C273.

[6] Manthiram A. and Goodenough J. B., Nature 329 (1987) 701.

[7] Wang X. Z., Henry M. and Livage J., J. Solid State Comm. 64 (1988) 881.

[8] Douy A. et OdIER P., brevet n 88-03371 et Mat. Res. Bull., soumis.

[9] Odier P., Dubois B., Gervais M. et Douy A., Mat. Res. Bull. 24 (1989). 11.

[10] Monod P., Dubois B. et Odier P., Physica C 153155 (1988) 1489.
[11] Dubois B., Ruffier D. et Odier P., J. Am. Ceram. Soc., sous presse.

[12] Roth R. S., Davis K. L. and Dennis J. R., $A d v$. Ceram. Mater. 2 (1987) 303.

[13] Frase K. G. and Clarke D. R., Adv. Ceram. Mater. 2 (1987) 295.

[14] Wang G., Hwu S. J., Song S. N., Ketterson J. B., Marks L. D., Poeppelmeier K. R. and MASON T. O., Adv. Ceram. Mater. 2 (1987) 313.

[15] De Leeuw D. M., Mutsaers C. A. H. A., LangeReIS C., SMOORENBURG H. C. A. and RoOMERS P. J., Physica C 152 (1988) 39.

[16] Dubois B., Douy A., Gervais M., Cabannes F. et Odier P., Physica C 153-155 (1988) 371.

[17] Greuter T., Kluge-Weiss P., ZimmermanN H. and SCHuler C., Phys. C 153-155 (1988) 361.

[18] Stucki F., Bruesch P. and Baumann Th., Physica C 156 (1988) 461. 